\title{
Intermittent Silent Coronary Spasm with Variant Premature Ventricular Contractions, and QRS-Complex Fragmentation in COVID-19 Pneumonia with Stroke and Pleural Effusion; Serious Risk but a Good Outcome
}

\author{
Yasser Mohammed Hassanain Elsayed* \\ Critical Care Unit, Kafr El-Bateekh Central Hospital, Egyptian Ministry of Health (MOH), Egypt
}

Submission: December 27, 2021; Published: January 24, 2022

*Corresponding author: Yasser Mohammed Hassanain Elsayed, Critical Care Unit, Kafr El-Bateekh Central Hospital, Damietta Health Affairs, Egyptian Ministry of Health (MOH), Damietta, Egypt

\begin{abstract}
Rationale: A pandemic COVID-19 virus is a multi-systemic lethal worldwide infection. Cardiac, neurological, chest, and renal systems are frequently involved. Silent coronary artery disease is sometimes reported in diabetic and elderly patients. There is a strong correlation between COVID-19 pneumonia, thromboembolism, and ischemic heart disease. Ischemic stroke and coronary artery disease are commonly clinically recognized in COVID-19 patients who have a risk impact on both morbidity and mortality. The QRS-complex fragmentation is considered as a marker for cardiac structural diseases inducing biventricular hypertrophy or any condition interfering with the normal homogeneous depolarization status inside the myocardium.
\end{abstract}

Patient concerns: A 68-year-old, non-working, smoker, married, Egyptian male patient was admitted to the intensive care unit with cerebrovascular stroke, premature ventricular contractions, and COVID-19 pneumonia.

Diagnosis: Intermittent silent coronary spasm with ischemic variant premature ventricular contractions, and QRS-complex fragmentation in COVID-19 pneumonia with stroke and pleural effusion.

Interventions: Electrocardiography, oxygenation, non-contrast chest $\mathrm{CT}$, and brain $\mathrm{CT}$.

Outcomes: Good response and better outcomes despite the presence of several remarkable risk factors were the results.

Lessons: Intermittent silent coronary spasm is an interesting issue and maybe multi-factorial. The presence of elderly male sex, heavy smoker, COVID-19 pneumonia, pleural effusion, recurrent ischemic cerebrovascular stroke, renal impairment, ischemic heart disease, hypocalcemia, diabetes, ischemic variant premature ventricular contractions, and QRS-complex fragmentations are prognostic factors for the severity of the disease. The clinical and electrocardiographic response after using anti-COVID19 measures the signifying its role and suggests the diagnosis of COVID19 infection.

Keywords: COVID-19 pneumonia; Intermittent silent coronary spasm; Premature ventricular contractions; Pleural effusion; QRS-complex fragmentation

Abbreviations: COVID-19: Coronavirus disease 2019; ECG: Electrocardiogram; ED: Emergency department; FQRS: Fragmented QRS; ICU: Intensive care unit; 02: Oxygen; PVCs: Premature ventricular contractions; SCD: Sudden cardiac death; SGOT: Serum glutamic-oxaloacetic transaminase; SGPT: Serum glutamic-pyruvic transaminase; SHD: Structural heart disease; VR: Ventricular rate

\section{Introduction}

Heart injury is commonly reported in coronavirus disease 2019 (COVID-19) cases. Indeed, up to $33 \%$ of

COVID-19 patients reveal electrocardiographic (ECG) ST-segment elevation despite angiographically there being normal coronary arteries [1]. An acute coronary syndrome (ACS) due to a coronary artery spasm (CAS) in a severe acute respiratory syndrome (SARS) coronavirus-2 patient [1]. Silent myocardial ischemia (SMI) is defined as objective documentation of myocardial ischemia in the absence of angina or anginal 
equivalents [2]. Patients with SMI were more likely to be elder, women, and diabetic [3]. Cardiac arrhythmias are significant leading causes of a high percentage of death among hospitalized COVID-19 patients [3]. The prognostic importance of silent ischemia is still controversial [2].

Although arrhythmias are uncommon in COVID-19 patients, they can be lethal which can be prevented by prompt recognition [4]. Premature ventricular contractions (PVCs) are a type of arrhythmia described as premature heart contractions and early depolarization of the myocardium originating in one of both ventricles due to an electrical impulse or ectopic rhythm from any part of the ventricles before the sinoatrial impulse has reached the ventricles [5]. Indeed, PVCs with structural heart disease (SHD) is considered a way to sudden cardiac death (SCD) [6]. The presences of PVCs in patients with ischemic heart disease (IHD) carry a poor prognosis6. Unfortunately, more-frequency PVCs carry a poor prognosis [6]. The presence of PVCs in patients with IHD is associated [6]. However, PVCs with established IHD may be viewed as a marker of disease severity with a poor prognosis [6].

Coronavirus disease 2019 (COVID-19) evolved quickly into a global pandemic with myriad systemic complications, including stroke. COVID-19 is an independent risk factor for stroke in hospitalized patients and mortality, and stroke presentations are frequently atypical [7]. Acute ischemic cerebrovascular accident (CVA) may happenin cases of coronavirus disease 2019 (COVID-19). The risk factors involving both in-hospital events and outcomes are still not well-been reported in large cohorts [8]. The QRScomplex fragmentation (fQRS) is the existence of high-frequency potentials or spikes in the QRS-complex [9]. The expression was first described in 1973 in reporting of an experimental study on canine hearts where coronary artery obstruction motivated the occurrence of fragmented ECG as a source of reentrant activity [10]. Previously, fQRS had been considered a hallmark for SHD triggering biventricular hypertrophy (BVH) [11].

The presence of fQRS can be elicited by any condition interrupting the normally homogeneous depolarisation status in the myocardium and causing regional conduction decelerating such as ischemia, scar, fibrosis, myofiber disarray, inflammation, and microvascular abnormality [9]. Das et al. revealed that there was a strong correlation between fQRS and the occurrence of myocardial scar in patients with coronary heart disease (CAD) in single-photon emission tomography (SPET) [12]. An incidence of pleural effusions is uncommon in COVID-19 [13]. Pleural effusion is considered an indicator for severe inflammatory state and carries poor clinical outcomes and might be an integral risk factor in critical COVID-19 infection [14].

\section{Case presentation}

A 68-year-old non-working, married male, Egyptian, heavy smoker patient was presented to the emergency department (ED) with tachypnea, left-side heaviness, acute confusion state, fever, and palpitations. Fatigue, irritability, dry cough, generalized body aches, anorexia, and loss of smell were the associated symptoms. There is a recent contact with a confirmed case of COVID-19 pneumonia. He was presented to the ED within 3 days of the above symptoms. He gives a history of old cerebrovascular stroke 3 years ago, diabetes on insulin, and hypertension on captopril. He is smoking about 30 cigarettes for 25 years. The patient denied a history of cardiovascular diseases, drugs, or other special habits.

Informed consent was taken. Upon general physical examination; generally, the patient was tachypneic, left hemiplegic, distressed, with an irregular pulse rate of VR; $80 \mathrm{bpm}$, blood pressure (BP) of $140 / 80 \mathrm{mmHg}$, respiratory rate of $22 \mathrm{bpm}$, the temperature of $37.9^{\circ} \mathrm{C}$, and pulse oximeter of oxygen $\left(\mathrm{O}_{2}\right)$ saturation of $94 \%$. There is left-side hemiparesis with GCS; 12 . Tests for latent tetany were elicited. Currently, the patient was admitted to the critical care unit with COVID-19 pneumonia, cerebrovascular stroke, and ischemic heart disease. Initially, the patient was treated with $\mathrm{O}_{2}$ inhalation by $\mathrm{O}_{2}$ cylinder $(100 \%$, by nasal cannula, $5 \mathrm{~L} / \mathrm{min})$. The patient was maintained treated with cefotaxime; (1000mg IV every 8hours), azithromycin (500mg PO single daily dose), oseltamivir (75mg PO twice daily only for 5 days), and paracetamol (500mg IV every 8 hours as needed).

SC enoxaparin $80 \mathrm{mg}$ twice daily), aspirin tablet (75mg, once daily), clopidogrel tablet (75mg, once daily), and hydrocortisone sodium succinate (100mg IV every 12 hours) were added. Furosemide IV amp (40mg, twice daily) was given. Intermittent hypotension had happened. Norepinephrine IVI $(0.8 \mathrm{mg} / \mathrm{ml}$, $3.8 \mathrm{ml} /$ hour ) was used. The patient was daily monitored for temperature, pulse, blood pressure, and $\mathrm{O}_{2}$ saturation. Serial ECG tracings through the hospital admission stay were done. The initial ECG tracing was done on the ICU admission of VR; 97 showing variant irregular PVCs, ST-segment depression in lateral leads (I and, aVL and V6), fragmentation QRS-complex in V1and 2 leads (Figure 1A).

The second ECG tracing was done within 8 minutes of the above tracing of VR; 98 showing variant irregular PVCs, fragmentation QRS-complex in V1 and 2 leads. There is a normalization of the above ST-segment depressions. There is a Wavy triple sign or Yasser's sign of hypocalcemia in aVL lead (Figure 1B). The third tracing was done within 8 hours of the above tracing of NSR of VR of 67 showing mild ST-segment depressions in lateral leads (V4-6), and fragmentation QRS-complex in V2 lead (Figure 1C). The fourth ECG tracing was done within 30 minutes of the above tracing of VR; 77 showings marked ST-segment depressions in both inferior (II and aVF) and lateral leads (V4 and 5), single PVC, Wavy triple sign, or Yasser's sign of hypocalcemia (V1 and V6 leads, and fragmentation QRS-complex in V2 lead (Figure 1D).

The fifth ECG tracing was done within 8 hours of the above tracing of NSR of VR of 76 showing normalization of the above abnormalities (Figure 1E). The initial brain CT without contrast was done within 11 days before hospital admission showing 
right ganglion infarction (Figure 2A). The first chest CT without contrast was done during the day of hospital admission showing mild bilateral ground-glass opacities with bilateral mild to moderate pleural effusion (Figure 2B). Currently, the patient was admitted to the critical care unit with COVID-19 pneumonia, cerebrovascular stroke, and ischemic heart disease. The initial complete blood count (CBC); Hb was $8.4 \mathrm{~g} / \mathrm{dl}$, RBCs; 3.14*103/ mm3, WBCs; 10.2*103/mm3 (Neutrophils; $81 \%$, Lymphocytes: 12\%, Monocytes; 7\%, Eosinophils; $0 \%$ and Basophils 0\%), Platelets; $143 * 103 / \mathrm{mm} 3$. S. Ferritin was high; $549 \mathrm{ng} / \mathrm{ml}$. D-dimer was high $(0.905 \mathrm{ng} / \mathrm{ml})$.

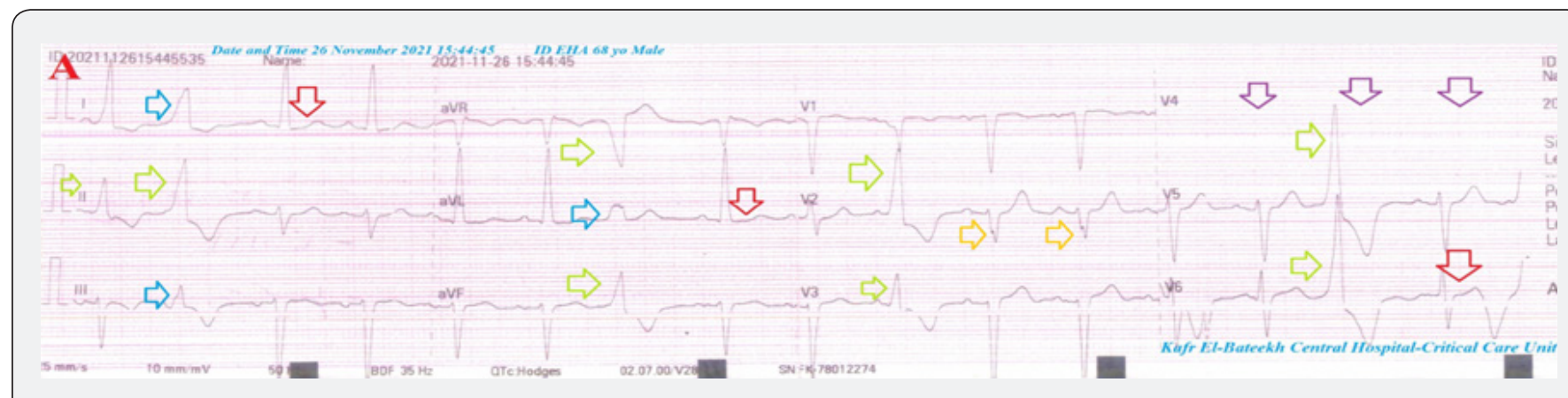

Figure 1A: Serial ECG tracings; A. tracing was done on the ICU admission of VR;97 showing variant irregular PVCs (light blue and lime arrows), ST-segment depression in lateral leads (I and, aVL and V6; red arrows), fragmentation QRS-complex in (V1 and 2; orange arrows). There is missed V4 lead (purple lines).

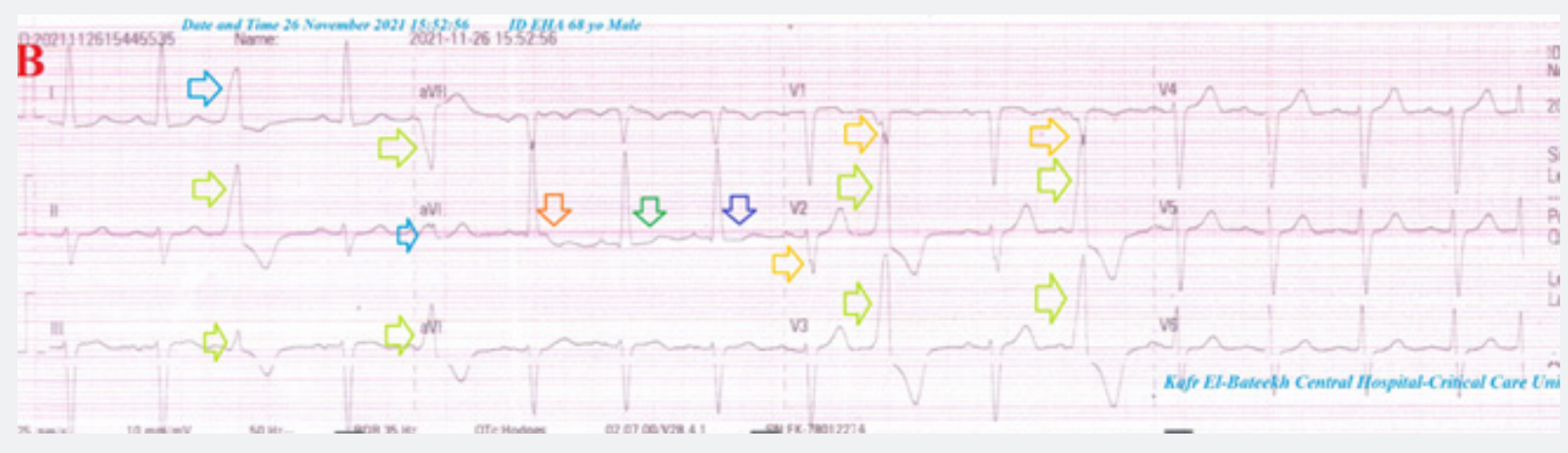

Figure 1B: Tracing was done within 8 minutes of the above tracing of VR; 98 showing variant irregular PVCs (light blue and lime arrows) and fragmentation QRS-complex in (V1 and 2; orange arrows). There is a normalization of the above ST-segment depressions. There is a Wavy triple sign or Yasser's sign of hypocalcemia (aVL lead; orange, green, and dark blue arrows).

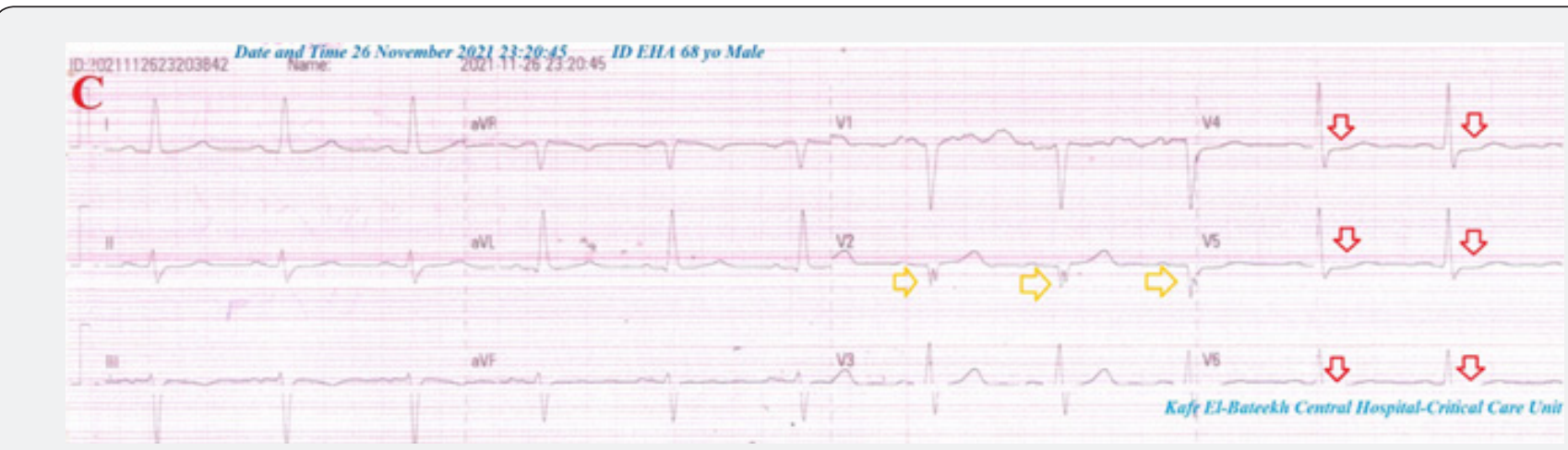

Figure 1C: Tracing was done within 8 hours of the above tracing of NSR of VR; 67 showing mild ST-segment depressions in lateral leads (V4-6; red arrows) and fragmentation QRS-complex in (V2; orange arrows). 


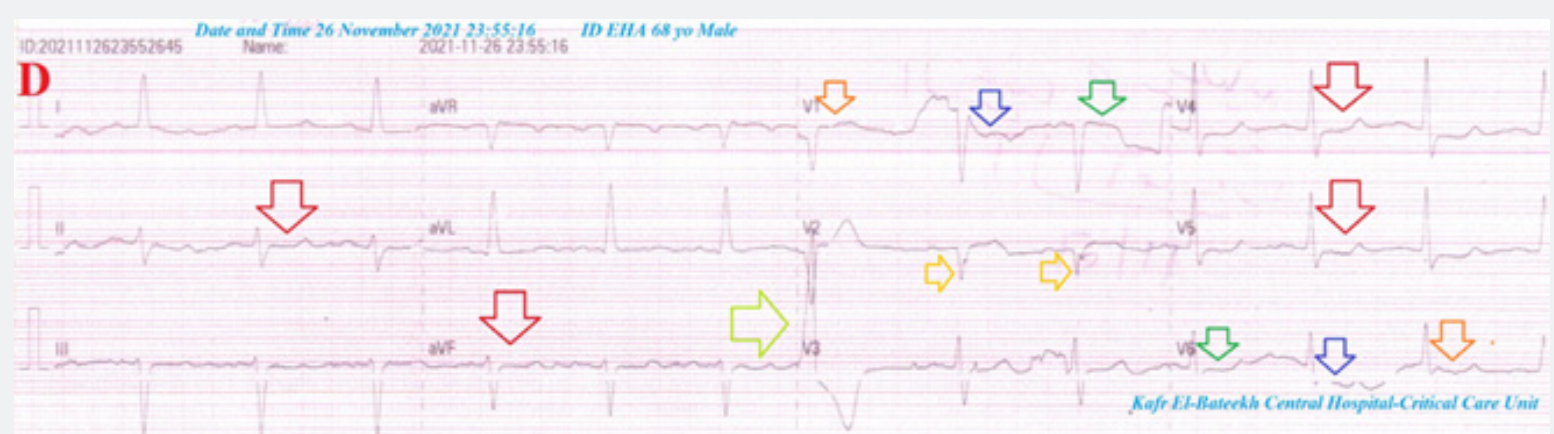

Figure 1D: Tracing was done within 8 hours of the above tracing of VR; 77 showed marked ST-segment depressions in both inferior (II and aVF; red arrows) and lateral leads (V4 and 5; red arrows), single PVC (lime arrow), Wavy triple sign or Yasser's sign of hypocalcemia (V1 and V6 leads; orange, green, and dark blue arrows), and fragmentation QRS-complex in (V2; orange arrows).

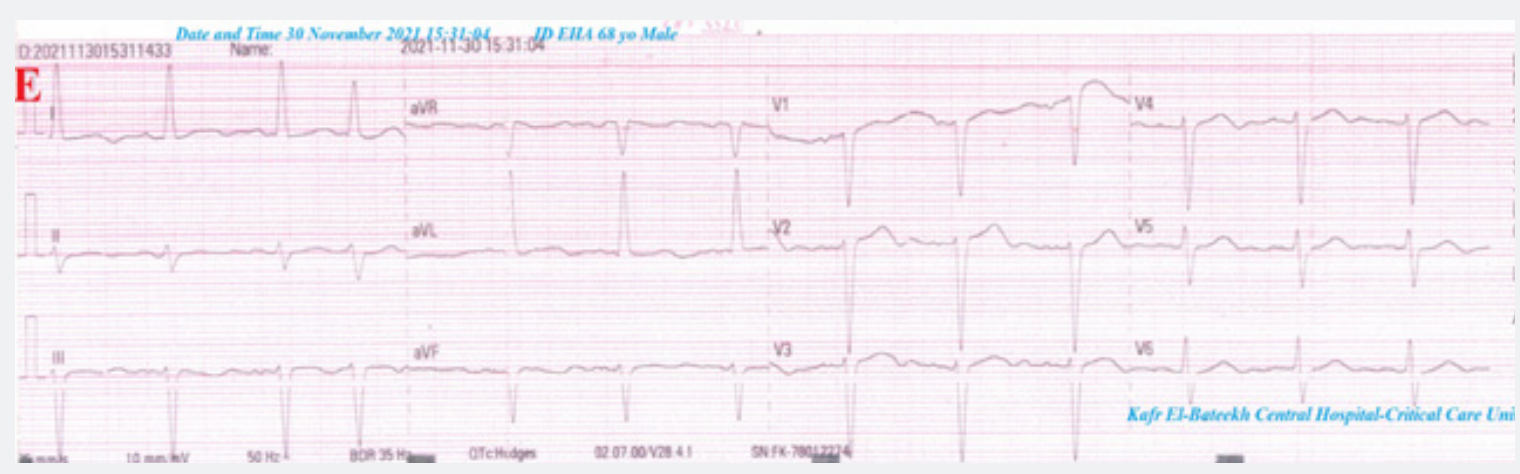

Figure 1E: Tracing was done within 8 hours of the above tracing of NSR of VR; 76 showing normalization of the above abnormalities.

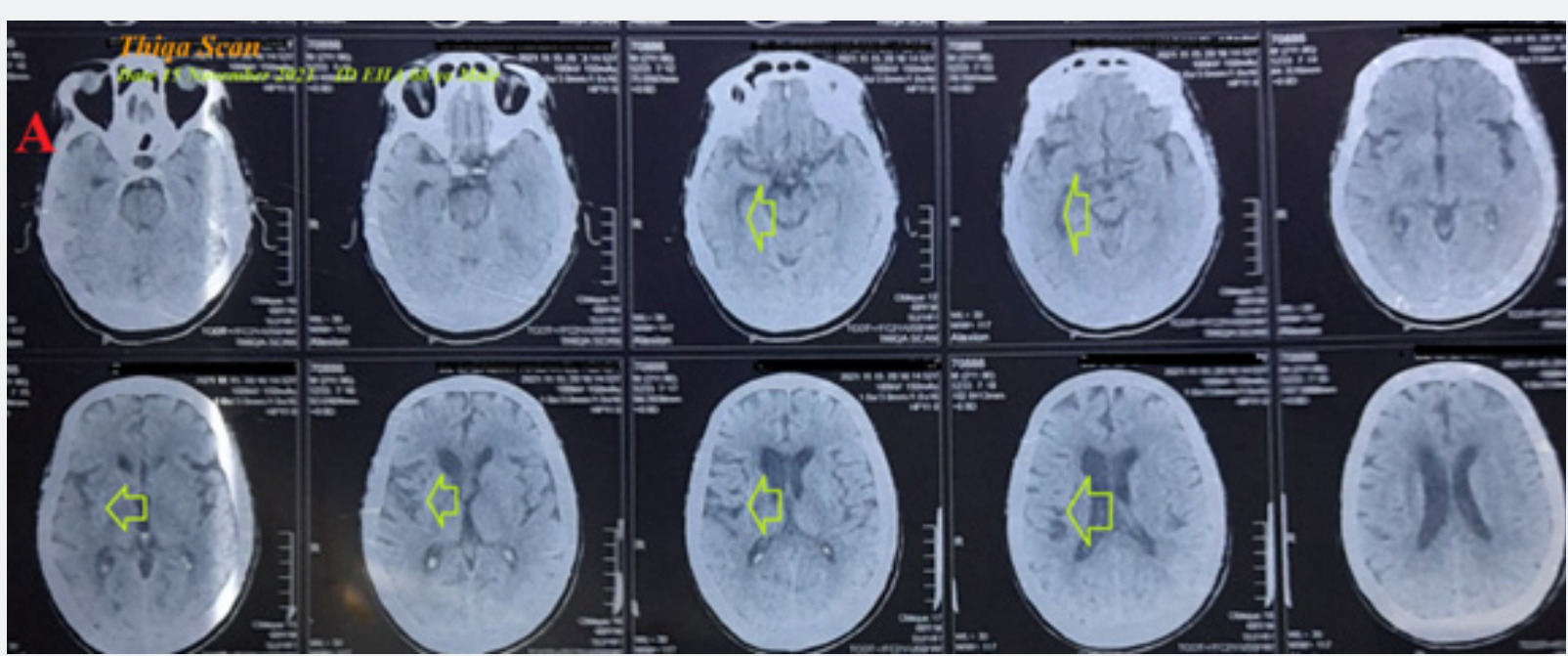

Figure 2A: Brain CT without contrast was done within 11 days before hospital admission showing right ganglion infarction (lime arrows)

CRP was high (35g/dl). LDH was high (512U/L). SGPT was normal (48U/L), SGOT was normal (47U/L). Serum albumen was low $(2.5 \mathrm{gm} / \mathrm{dl})$. Serum creatinine was high $(2.1 \mathrm{mg} / \mathrm{dl})$ and blood urea was high (86mg/dl). PTT was normal (31.6 seconds). RBS was normal (165mg/dl). Plasma sodium was normal (143mmol/L). Serum potassium showed hypokalemia $(3.1 \mathrm{mmol} / \mathrm{L})$. Ionized calcium was low $(1.06 \mathrm{mmol} / \mathrm{L})$ and total calcium was low $(7.4 \mathrm{mg} / \mathrm{dl})$. The troponin test was negative $(0.01 \mathrm{U} / \mathrm{L})$. CK-MB was normal $(11 \mathrm{U} / \mathrm{L})$. Brain CT without contrast was done during the day of hospital discharge showing old right ganglion infarction and recent lacunar infarctions (Figure 2C). 


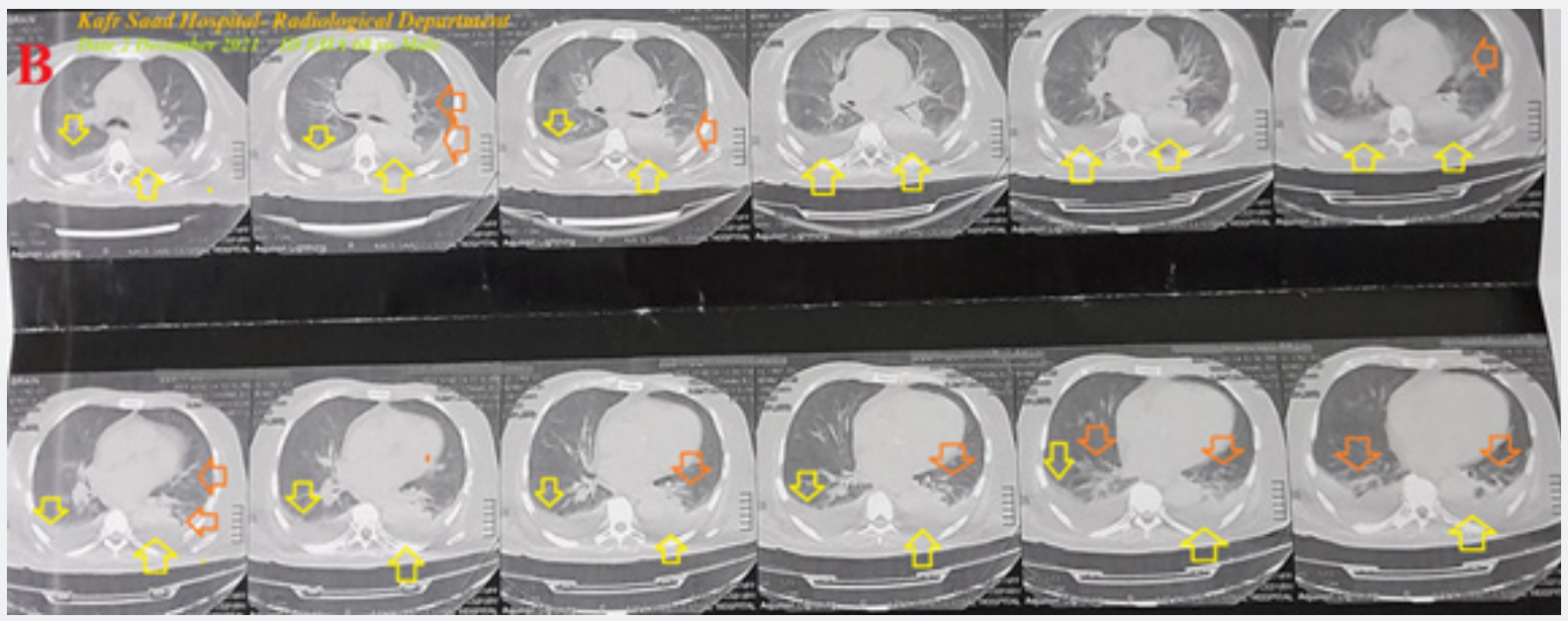

Figure 2B: Chest CT without contrast was done during the day of hospital admission showing mild bilateral ground-glass opacities (orange arrows) with bilateral mild to moderate pleural effusion (yellow arrows).

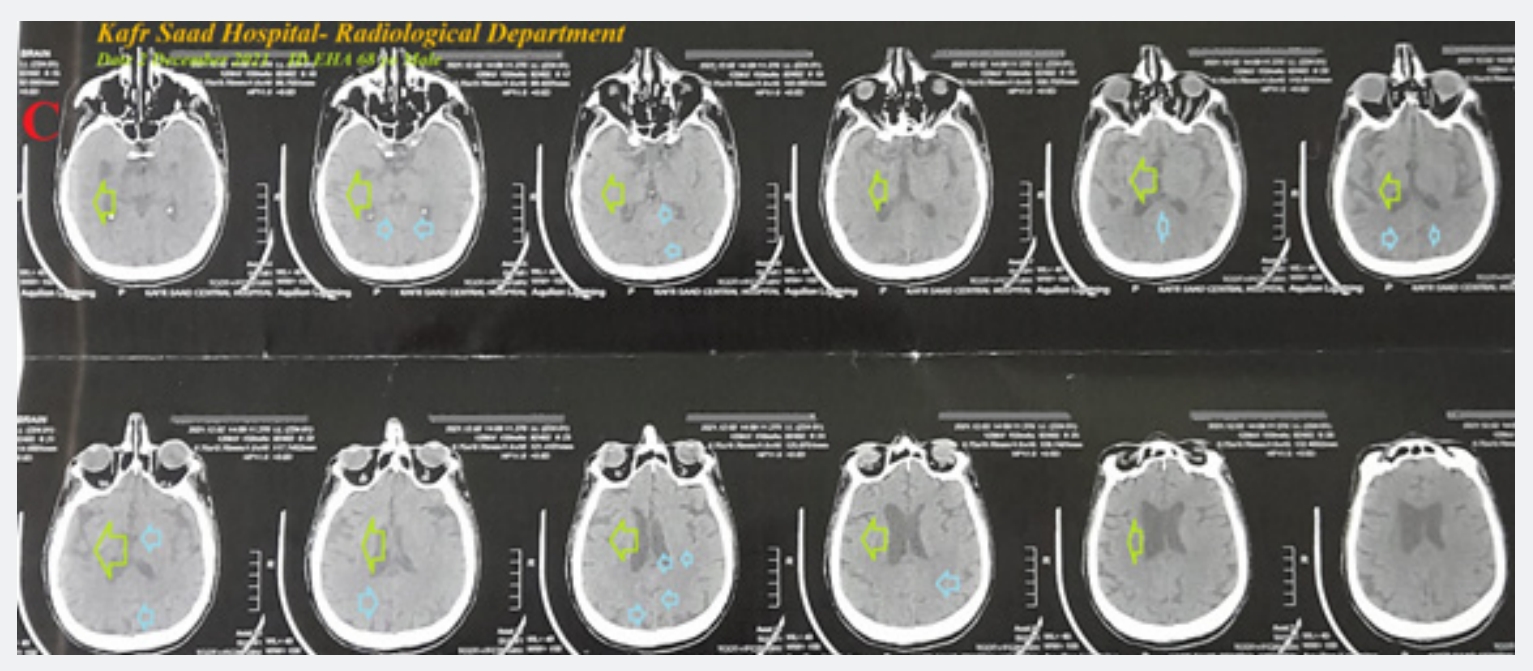

Figure 2C: Brain CT without contrast was done during the day of hospital discharge showing old right ganglion infarction (lime arrows) and recent lacunar infarctions (light blue arrows).

Later echocardiography showied mild inferolateral hypokinesia with an EF of 54\%. COVID-19 pneumonia, ischemic heart disease, and stroke were the most probable diagnosis. The patient was discharged within 8 days of hospital admission nearly, after clinical, electrocardiogram, and workup improvement. The patient was continued on aspirin tablet (75mg, OD), long-acting nitroglycerine oral capsules (2.5mg BID), furosemide oral tablets (40mg fasting OD), insulin mixtard (70/30, 10/5, BID), captopril (12.5mg, OD), and calcium with vitamin D oral preparations (OD) for 2 weeks with follow-up. Further cardiac, neurological, and chest follow-up was advised.

\section{Discussion}

\section{Overview}

A 68-year-old, non-working, smoker, married, Egyptian male patient was admitted to the intensive care unit with cerebrovascular stroke, premature ventricular contractions, and COVID-19 pneumonia.

I. The primary objective for my case study was the presence of a patient who presented with intermittent silent coronary artery spasm with ischemic variant premature ventricular contractions, and QRS-complex fragmentation in COVID-19 pneumonia with stroke and pleural effusion in the ICU.

II. The secondary objective for my case study was the question of; how did you manage the case at home?

III. There was a history of contact with a confirmed COVID-19 case. The presence of confirmed COVID-19 case, bilateral ground-glass consolidation, and laboratory COVID-19 suspicion on top of clinical COVID-19 presentation will strengthen the COVID-19 diagnosis. 
IV. Interestingly, intermittent hypotension, with COVID-19 pneumonia with stroke and pleural effusion plays an essential role in the present intermittent silent coronary artery spasm.

V. The presence of fragmentation of the QRS-complex is considered another risk.

VI. Elderly male sex, heavy smoker, COVID-19 pneumonia, pleural effusion, recurrent ischemic cerebrovascular stroke, renal impairment, ischemic heart disease, hypocalcemia, diabetes, ischemic variant premature ventricular contractions, and QRScomplex fragmentation are risk factors.

VII. Acute myocardial infarction was the most probable electrocardiographic differential diagnosis for the current case study. but the ST-segment elevation is always seen in all beats of at least two consecutive leads.

VIII. I can't compare the current case with similar conditions. There are no similar or known cases with the same management for near comparison.

IX. The only limitation of the current study was the unavailability of coronary catheterization.

\section{Conclusion and Recommendations}

i. Intermittent silent coronary spasm is an interesting issue and maybe multi-factorial.

ii. The presence of elderly male sex, heavy smoker, COVID-19 pneumonia, pleural effusion, recurrent ischemic cerebrovascular stroke, renal impairment, ischemic heart disease, hypocalcemia, diabetes, ischemic variant premature ventricular contractions, and QRS-complex fragmentations are prognostic factors for the severity of the disease.

iii. The clinical and electrocardiographic response after using anti-COVID19 measures the signifying its role and suggest the diagnosis of COVID19 infection.

\section{Acknowledgement}

I wish to thank the team nurses of the critical care unit in Kafr El-Bateekh Central Hospital who make extra-ECG copies for helping me. I want to thank my wife to save time and improving the conditions for supporting me.

\section{References}

1. Rivero F, Antuna P, Cuesta J, Alfonso F (2021) Severe coronary spasm in a COVID-19 patient. Catheter Cardiovasc Interv 97(5): E670-E672.

2. Peter FC, Kim MF, and With the Assistance of, and Caroline Daly (2003) Silent Myocardial Ischemia. Circulation 108(10): 1263-1277.

3. Goldman L, Kirtane A (2003) Triage of Patients with Acute Chest Pain and Possible Cardiac Ischemia: The Elusive Search for Diagnostic Perfection. Ann Intern Med 139(12): 987-995.

4. Mohammadyari E,, Ahmadi I, Mohammadyari A, Tavan H, Norozi S (2021) The frequency of arrhythmias in COVID-19 patients, a study in the Shahid Mostafa Khomeini Hospital of Ilam from March to August 2020. New Microbes New Infect 41: 100867.

5. Elsayed YMH (2019) Test the Termination of Regular Ischemic Premature Ventricular Contractions Variants with Nitroglycerin; A new Therapeutic and Diagnostic Test; Retrospective Observational Study (14-Report Cases). Emerg Med Trauma Care J.

6. Elsayed YMH (2019) Premature Ventricular Contractions from Benign to Seriousness - A Narrative Updating Review. Archives of Emergency Medicine and Intensive Care 2(2): 1-21.

7. Katz JM, Libman RB, Wang JJ, Sanelli P, Filippi CG, et al. (2020) Cerebrovascular Complications of COVID-19. Stroke 51(9): e227-e231.

8. Qureshi AI, Baskett WI, Huang W, Shyu D, Myers D, et al. (2021) Acute Ischemic Stroke and COVID-19: An Analysis of 27676 Patients. Stroke 52(3): 905-912.

9. Brohet C (2019) Fragmentation of the QRS complex: the latest electrocardiographic craze?. Acta Cardiol 74(3): 185-187.

10. Boineau JP, Cox JL (1973) Slow ventricular activation in acute myocardial infarction. A source of re-entrant premature ventricular contractions. Circulation 48(4): 702-713.

11. Flowers NC, Horan LG, Thomas JR, W J Tolleson (1969) The anatomic basis for high-frequency components in the electrocardiogram. Circulation 39(4): 531-539.

12. Das MK, Khan B, Jacob S, Awaneesh K, Jo M (2006) Significance of a fragmented $Q R S$ complex versus a $Q$ wave in patients with coronary artery disease. Circulation 113(21): 2495-2501.

13. Ng MY, Lee EYP, Yang J, Fangfang Y, Xia L, et al. (2020) Imaging Profile of the COVID-19 Infection: Radiologic Findings and Literature Review. Radiol Cardiothorac Imaging 2(1): e200034.

14. Zhan N, Guo Y, Tian S, Binglu H, Xiaoli Tian, et al. (2021) Clinical characteristics of COVID-19 complicated with pleural effusion. BMC Infect Dis 176. 
Your next submission with Juniper Publishers will reach you the below assets

- Quality Editorial service

- Swift Peer Review

- Reprints availability

- E-prints Service

- Manuscript Podcast for convenient understanding

- Global attainment for your research

- Manuscript accessibility in different formats ( Pdf, E-pub, Full Text, Audio)

- Unceasing customer service

Track the below URL for one-step submission https://juniperpublishers.com/online-submission.php 